\title{
¿Emprendimiento moral en la identidad chilena? Análisis sociológico sobre clases sociales y televisión
}

\author{
口曰 \\ JOSÉ IGNACIO NAZIF \\ Universidad de Chile
}

\begin{abstract}
::: RESUMEN
El presente artículo busca contribuir al análisis de la identidad chilena y la televisión. En oposición a estudios que señalan que la función de emprendimiento moral está ubicado en las clases altas, y que las clases bajas carecen de éste, se sostiene a través de un análisis empírico lo siguiente; primero, la clase alta, de acuerdo al concepto de tradicionalismo ideológico, sólo en la esfera privada tiene patrones conductuales que pueden relacionarse a esta función social. Segundo, para el caso de lasclases bajas, y retomando el concepto de conciencia dual sugerido por Fanon, la función de emprendimiento moral se vincula fuertemente a la esfera pública como una estrategia de blanqueamiento (o de adaptación) de su discurso.Tercero, la clase baja, a diferencia de la clase alta, está en mayor sintonía con la percepción de que el consumo de la televisión provoca efectos negativos en los televidentes. Se sugiere, por una parte, revisar el concepto de emprendimiento moral, dado que habría un cambio de orden práctico respecto a como esta función se estaría ejecutando para el caso de la televisión, y por otra, ahondar sobre los mecanismos sociales que inhiben o facilitan la función de emprendimiento moral.
\end{abstract}

PA LA B R A S C LAVE : emprendimiento moral, televisión, clases sociales

\section{::: ABSTRACT}

This article contributes to the analysis of $C$ hilean identity and television. There are studiesw wich point out that the moral entrepreneurship function is a constitutive part of the high class, and they also show that the lower class lacks it completely. U nlike these studies it is argued, through an empirical analysis, the following: Firstly; the high class, according to

* Q uisiera agradecer al C onsejo $\mathrm{N}$ acional deTelevisión por facilitarme la base de datos de la Encuesta $\mathrm{N}$ acional deTelevisión 2005. También agradezco a Aldo M ascareño y Patricio $\mathrm{N}$ avia por susimportantes colaboraciones en versiones anteriores de este artículo. La responsabilidad por el análisise interpretación realizada es exclusivamente mía. 
ideological traditionalism, displays moral entrepreneurship behaviors only at the private sphere. Secondly, the lower class, according to the dual conscience concept suggested by Fanon, performsa moral entrepreneurship function at the public sphere as a strategy to whitening its discourse.T hirdly, unlike high class people, members of lower class believe that television consumption causes negative effects in its audience. Since there is a change on how this function is performed when the television is the focus of analysis, it is suggested to theoretically review the concept of moral entrepreneurship. Second, it also proposed to deepen the analysis of which social mechanisms inhibit or trigger the function of moral entrepreneurship.

K EY W O R D S: moral entrepreneur, television, social classes

\section{Introducción}

A partir del reestablecimiento de la democracia en $\mathrm{C}$ hile, diversos estudios han enfocado su análisis sobre la identidad sociocultural de la sociedad chilena. Las áreas específicas de estos análisis han sido diversas: reidentificación de clases sociales, nuevas élites, enclaves autoritarios, consumismo, modernización, movilidad social, medios de comunicación (M oulian, 1997; PN U D, 1998; Tironi, 1999; Larraín, 2001;Torche y Wormald, 2004;A tria, 2004; C astells, 2005; M arín y Cordero, 2005; Torche, 2005). N o obstante el amplio espectro de estos estudios, es necesario seguir profundizando en algunas de estas áreas para ampliar el conocimiento sobre este proceso. Específicamente, estudios sobre la relación identidad chilena y televisión han sido escasos. En esta línea destacan el trabajo deT ironi y Sunkel (1993), por una parte, y el de M arín y Cordero (2005) por otra. En el primero se enfatiza la relación democracia y medios de comunicación, mientras que en el segundo se identifica la expansión del proceso de deliberación democrática facilitada por la irrupción de los medios masivos. A partir de un análisis empírico, este artículo busca contribuir a la discusión sobre la identidad chilena y su relación con la televisión. Para ello se considerará como objeto de análisis las características de la identidad chilena a partir de la conducta que las clases sociales han desarrollado.

Se destaca de la identidad chilena la siguiente contradicción: i) equiparar casi en su totalidad a la clase alta con funciones de moral entrepreneur (M oulian, 1997; T ironi, 1999; Velasco, 2001; PN U D, 2004; R ovira, 2005; M olina, 2004; sobre el concepto de moral entrepreneur. Becker, 1963), es decir, paternalistas, con un fuerte sentido de lo que es correcto y dispuestos a ejercer un control en coherencia a sus valores; y ii) entender a la clase popular como un grupo cuya matriz cultural se caracteriza por tensiones e incoherencias, donde priman las transgresiones y las acciones libertarias, y por tanto lejos de ejercer funciones de emprendimiento moral (Salazar, 1991; Salazar y Pinto, 1999).

Considerando esta diferencia cultural, se podría señalar que ésta también se reproduce cuando se analiza la relación clase-televisión. En primer término, si la televisión es asimilada a una noción ideal de esfera pública como la define 
$\mathrm{H}$ abermas, y recogiendo la tesis planteada por M arín y Cordero (2005), en la elite se podría encontrar mayores disposiciones a aprobar mecanismos de censura, mientras que para aquellos representantes de las clases populares éstos serían menos rígidos. En segundo lugar, si la televisión fuese considerada fuente efectiva de conductas negativas para los miembros de la sociedad, la clase al ta estaría prácticamente en línea con juicios que operacionalicen dicha expresión, mientras que los representantes de las clases populares en una posición contraria. En tercer término, y tomando como representación de la esfera privada el ejercicio del control sobre los menores, la clase alta tendería a ser más estricta que la clase baja.

En este artículo se señalará a través de un análisis empírico que esta contradicción está invertida para las dimensiones (i) esfera pública y (ii) efectos de la televisión, y sólo es válida para la (iii) esfera privada. En consecuencia, las tres tesis aquí sugeridas son las siguientes: (i) Cuando la televisión es entendida como esfera pública, a diferencia de la clase alta, las clases bajas despliegan una función de emprendimiento moral, porque sugieren controles menos flexibles. (ii) La elite ejerce una función de emprendimiento moral más laxa que los representantes de la cultura popular, cuando sus respectivos juicios respecto a los efectos negativos de la televisión son comparados. (iii) Sólo en la esfera privada el orden de la contradicción cultural se mantiene, es decir, la clase alta ejercería su función de emprendimiento moral puesto que los adultos de esa clase supervisarían mayormente, en forma directa e indirecta, lo que los menores del hogar ven. $\mathrm{D}$ ado que dichos resultados son bastantes novedosos, se propondrá como marco explicativo el desempolvamiento de dos conceptos, «tradicionalismo ideológico» y «conciencia dual». C ada uno de éstos será aplicado a las clases alta y baja respectivamente.

El artículo está dividido en cuatro partes. En la primera, se ahondará específicamente sobre el funcionamiento social que cumplen los emprendedores mora les. Seguidamente, y según lo propuesto por algunos estudios y autores, se esta blecerá la relación de las clases sociales alta y baja con dicha característica. En una segunda parte se realizará una operacionalización funcional del concepto de moral entrepreneur, el cual permitirá abrir las dimensiones sobre el cual se hará el análisis empírico. En la tercera parte se explicará la metodología a aplicar, la cual se basa en la utilización de regresiones lineales, logística-multinomial y binaria. Finalmente serán discutidos los resultados obtenidos en torno a dos elementos: primero, con relación a las tesis (i), (ii) y (iii) aquí propuestas, y segundo, respecto a la necesidad teórica de extender el concepto de emprendimiento moral, dado que habría un cambio de orden práctico respecto a como esta función se estaría llevando a cabo para el caso de la televisión.

\section{Emprendedores morales en Chile: $\mathrm{R}$ evisando la contradicción clase alta y clase popular}

Para entender parte esencial de la identidad chilena y su relación con la televisión, resulta fundamental discutir la contradicción sociocultural que hay entre 
las clases alta ${ }^{6}$ y baja. Para ello se tomará como eje teórico el concepto de moral entrepreneur, el cual permitirá distinguir la función que ambos grupos sociales podrían estar cumpliendo respecto de la televisión.

\section{1) LA FUNCIÓN DE LOS EMPRENDEDORES MORALES}

El concepto de moral entrepreneur fue acuñado por H oward Becker (1963). Dicho autor señala que dicho grupo se caracteriza por crear y recrear reglas, normas, leyes y valores. A través de actitudes paternalistas sus miembros utilizan los diversos recursos públicos y privados para poder implementar satisfactoriamente sus programas 0 agendas morales. Para reforzar el logro de dichos objetivos estos grupos aprovechan las atmósferas social es de pánico, puesto que ahí ciertas normas y leyes tienen mayores probabilidades de ser implementadas e institucionalizadas (Becker, 1963). Es característica propia de este tipo de grupos desarrollar un fuerte sentido identitario, el cual facilita la distinción con aquellos grupos que representan las desviaciones en torno a lo apropiado. ${ }^{9}$

$\mathrm{H}$ ay dos funciones de este tipo de grupos que interesa destacar: (i) Dicho grupo intenta crear normas. Precisamente un emprendedor moral no siente que las normas bajo las cuales la sociedad funciona sean suficientemente fuertes para eliminar las conductas inapropiadas o a los grupos desviados. C onsecuentemente dichos grupos sienten la responsabilidad de corregir ciertos problemas sociales para proteger a la sociedad de las inmoralidades (B ecker, 1963). (ii) La segunda función está asociada a la fiscalización de las normas, es decir, menos interesado en el contenido de éstas que sobre la observación de su correcto cumplimiento. C on relación a esta última característica están aquellas personas cuya identidad profesional está basada en la necesidad social de ejercer la vigilancia en distintos ámbitos, i.e., policías, inspectores de colegios y/ 0 auditores. Estos emprendedores morales necesitan justificar sus profesiones, puesto que creen

\footnotetext{
${ }^{6}$ A un cuando se reconoce la diferencia conceptual propuesta por W right M ills (1956) entre élite y clase alta, en este trabajo ambos conceptos serán utilizados al ternadamente. Lo anterior debido a que para el caso chileno dicha distinción es claramente problemática. En primer lugar,Torche (2005) señala que la movilidad desde la clase más alta (del esquema de clases de Erikson y G oldthorpe) hacia otros estratos es muy baja. En segundo término, una investigación realizada por el PN UD (2004), destaca un grado importante de rigidez en la sociedad chilena, puesto que se aprecia una muy baja probabilidad de que una persona con orígenes socioeconómicos medios y bajos acceda a la clase alta. Esto sin embargo, y como se verá más adelante en nota 12 , no significa señal ar que haya un discurso homogéneo en dicha clase. Sino, à la Bourdieu, más bien la existencia de una contradicción entre nueva y vieja burguesía (ver Bourdieu, 1997).

${ }_{9}$ Es importante recalcar que el sentido de identidad no se limita al acceso de recursos materiales o culturales, es decir, la definición de moral entrepreneur no está supeditada particularmente a clases sociales o socioeconómicas, puesto que también resalta el carácter religioso que este grupo podría tener.Vale la pena señalar que los moral entrepreneursno son en sí conservadores, machistas o autoritarios, es decir, no hay una identificación fija respecto de dicho grupo, ya que en dependencia al contexto estos también pueden ser liberales, feministas y democráticos.
} 
que su correcta función produce impactos positivos en la sociedad o en organizaciones sociales (G oode y Ben-Yehuda, 1999).

\section{II) EM PR EN DIMIENTO MORAL EN LAS CLASES ALTA Y BAJA CHILENAS}

Para poder determinar la función de emprendimiento moral en las clases sociales, se analizará una serie de estudios. Por un lado serán revisados aquellos que señalan que dicha función es exclusiva de la clase alta, y por el otro, aquellos que argumentan que ésta puede ser ejercida por ambas, y por tanto el énfasis debe estar puesto en los ámbitos de acción social esfera pública y esfera privada.

$R$ especto de la clase alta, ${ }^{12}$ en primer lugar se destaca Tironi, quien señala que desde los años noventa, dicho grupo, incluyendo por cierto en éste representantes de la izquierda política, se siente amenazado por la aparición abrupta de las masas. Es decir, él entiende que a partir del proceso de democratización chileno, la clase alta percibe que el sistema social puede tornarse anómico, y en consecuencia «la clase alta] se plantea a nombre y por la gente [y especialmente con la responsabilidad de] disciplinar las expectativas y constreñir los grados de libertad individual» (T ironi, 1999: 53). Está escondido ahí un reclamo aristocrático, puesto que dicho proceso permite aperturas que llevan a cambios de ciertas normas sociales, que no necesariamente son coherentes con aquellas que la clase alta considera apropiadas para el país.

U n segundo tipo de estudio en esta línea es aquel desarrollado por el PN U D (2004: 172-92). En éste se describe la composición interna de la elite en cuatro tendencias: Progresistas Perplejos, C onservadores, Liberales G lobalizados y Liberales Progresistas. Sin embargo, dichos grupos tendrían algo en común, sostienen los investigadores del PN U D. En la elite se comparte la noción de que el «otro no elite» debe ser tutelado, dada su nula capacidad para autoconducirse. En suma, más allá de la heterogeneidad que dicha clase pueda mostrar en términos políticos, ésta tiene una matriz cultural transversal cuya característica principal es ser emprendedores morales.

U n tercer y último estudio cuyas conclusiones apuntan en una dirección similar es aquel elaborado por M arín y Cordero (2005). Vale la pena señalar, sin

\footnotetext{
${ }^{12}$ Vale la pena recordar tres aspectos sobre la clase alta: históricos, de estructura interna y políticos. Aun cuando a partir de los años setenta se observa que la clase al ta chilena muestra una diversificación en términos económicos y cambia su discurso en torno al rol que el Estado debía jugar en la economía, su carácter de clase permanece (ver K irsch, 1977; y T ironi,A güero y Valenzuela, 2001). Segundo, internamente se puede reconocer una composición proveniente directamente del mundo empresarial privado, y una segunda, o una new elite, que básicamente funciona a partir del nicho generado al interior de los poderes ejecutivo y legislativo. C ada una de estas tiene un referente político siendo la primera asociada a la derecha, y el segundo a la centro-izquierda (verVelasco, 2001; R ovira, 2005).Tercero, Blofield (2002) y D ávila (2002) señalan que la derecha política y la centro-izquierda presentan rasgos conservadores y liberales. C abe precisar, sin embargo, que ninguna de estas fuerzas recorre por completo el espectro ya que la primera no llega al extremo de la izquierda, ni la segunda al extremo de la derecha.
} 
embargo, que éste está enfocado sobre la elite política, y por tanto no necesariamente se vincula directamente a la clase alta per se. Estos autores señalan que dicho grupo está disconforme con respecto a cómo la agenda y estilo de los medios de comunicación han sido desarrollados en el último tiempo, pero es de particular importancia relevar que los contenidos introducidos son para dicho grupo banaleso de poca importancia para el país. Es precisamente esta capacidad de saber qué es importante para el país lo que hace que dicho grupo pueda ser entendido como emprendedor moral.

Con respecto a la clase baja, los estudios analizados tienen una fuerte resonancia teórica en Elias (1994). De la contradicción civilización/ barbarie, algunos autores toman esta última característica para sintetizar la cultura popular. En primer término, Salazar señala que esta versión de la cultura está en directa oposición a la cultura oligárquica de la elite. Precisamente, destaca Salazar (1991), los miembros de la cultura popular han desarrollado acciones incoherentes, llena de tensiones las cuales le imprimen a ésta un sello creativo e innovador. Bajo este precepto, Salazar también observa que representantes de dicha cultura tendrían una conducta errática respecto de cómo introducirse a definir reglas, normas y leyes. Es decir, sería difícil reconocer en dicho grupo la realización de un trabajo sistemático, «consciente», que haya permitido institucionalizar oficialmente ciertos preceptos. Al contrario, la serie de acciones que ellos han institucionalizado está más bien asociada a irrupciones aleatorias de actos violentos, que «han remecido por entero la estabilidad del sistema» (Salazar, 1990: 13).

0 tro trabajo que hace una revisión en estos términos es aquel correspondiente a Peppelenbos (2005). Dicho autor entiende que la cultura popular, situada en un contexto institucionalizado de relaciones patrones/ clientes, también tiene rasgos muy heterogéneos en su interior. De ésta se destaca formas débiles de corporativismo, fuerte fatal ismo, ocasionales rebeliones so rdas, y a diferencia de Salazar, él entiende que ésta no promueve acciones de carácter innovador, puesto que las acciones están enmarcadas en un apego formal a lo acordado, en «no hacer más de la cuenta» (Peppelenbos, 2005). Es decir, esta matriz cultural no produciría acciones dentro de los canales oficiales cuyos objetivos fueran desbaratar, o poner seriamente en jaque la legalidad de ciertas costumbres, puesto que no existe la noción de un orden superior a ser defendido. Tampoco, dicha cultura facilitaría la formación de grupos, o profesiones, cuya función fuese insistir en la perentoriedad de seguir controlando ciertos ámbitos sociales.

En la figura 1 se muestra cómo estaría desplegada la función de moral entrepreneur para las clases sociales de acuerdo a los trabajos recién revisados. Como se puede apreciar el endurecimiento o desaparición de la función de emprendimiento moral está asociada a la membresía de clase alta y baja respectivamente. No obstante lo anterior, hay dos versiones que merecen mayor detención porque, dicha función no necesariamente se daría en la dirección anteriormente descrita.

En primer término, se destaca lo que señala Germani (1981: 169) como «tradicionalismo ideológico». Dicho concepto intenta capturar los cambios in- 


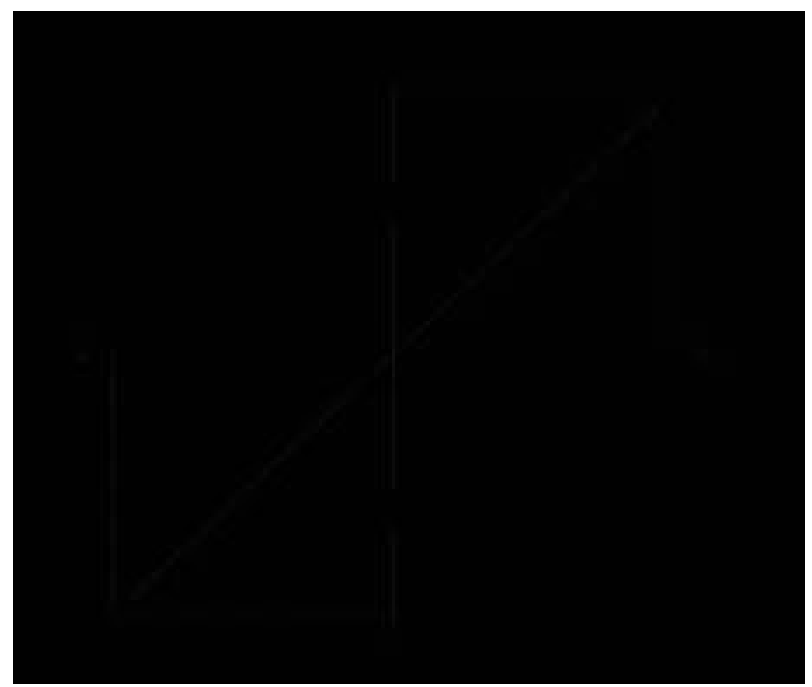

Figura 1. Caracterización teórica del funcionamiento del emprendimiento moral con relación a las clases sociales

tensos que viven las elites en los países en desarrollo. Particularmente respecto al proceso de desarrollo, estos grupos tienen una mirada ambivalente, puesto que aun cuando no lo rechazan, promueven y aceptan ciertos aspectos de éste. Por una parte apoyan cambios en el área económica, siempre y cuando estas transformaciones sean la base de la independencia del país, y por otra, rechazan los cambios asociados a dicha transformación que perturben el funcionamiento de instituciones «tradicionales» tales como la familia, la educación, y la estratificación social.

Dicho autor enfatiza que no son los contenidos en sí mismos los que definen al tradicionalismo ideológico, sino más bien a la opción que los grupos toman conscientemente ante una situación controversial en el seno de la sociedad. Al haber la elite estado sujeta a un proceso de elección, el contexto social donde ésta está ubicada pudo también haber sufrido cambios traducidos éstos en las actitudes de sus miembros. Precisamente en dicha situación la elite puede emprender un rol vanguardista, y la dirección innovadora o conservadora está finalmente sujeta a sus propios intereses, y como se dijo anteriormente, no a valores en sí mismos (Germani, 1981; Lechner, 1999: 3). De lo anterior se puede desprender que la elite puede dejar de lado la función de moral entrepreneur en ciertos ámbitos, especialmente en aquellos que estén ligados al desarrollo económico. En consecuencia, el mismo avance de la televisión puede ser aceptado siempre y cuando ésta reafirme ciertos procesos económicos y no afecte a la familia o a la educación. Para esto último la elite ejecutaría estrategias de control adhoc en la esfera privada.

En segundo término está una noción que Fanon implícitamente desarrolla para analizar el racismo en sociedades colonizadas, el cual puede ser llamado «conciencia dual». Éste puede ser aplicado a la clase baja, puesto que como 
señala Larraín en C hile la «estratificación social, aun aquella de carácter capitalista, siempre ha ido acompañada de un elemento racial: en Chile, de manera general, mientras más oscura la piel más baja la clase social» (2001: 232).

Dicho concepto puede ser desprendido del ensayo «Peau noire, masquesblancs» (Fanon, 1971). En éste, Fanon intenta describir como el racismo está relacionado con las actitudes que finalmente desarrollan los «negros» en contextos sociales donde ha habido procesos de colonización. Éste autor señala que la identidad del «negro» se ha construido en relación con el «blanco», y dada que ésta se ubica en un polo de dominación, el «negro» finalmente se effuerza por asimilar los valores dominantes. En consecuencia, argumenta Fanon y con una clara influencia de la teoría de alienación marxista, los valores culturales asociados al grupo dominante son internalizados en la conciencia y producen finalmente un quiebre entre la conciencia del «negro»y su propio cuerpo. Evidentemente esto implica que la identidad «negra» se ha alienado de sí misma (Fanon, 1971: 17). Por otra parte, vale la pena señalar que la identidad social del «blanco» también es construida en referencia al negro, aun cuando ésta ocupe el lugar del dominado. Por tanto la identidad cultural de una sociedad determinada necesariamente se va produciendo a través de la interacción de ciertos grupos, teniendo como resultados mixturas en distintos ámbitos y no características inmutables. ${ }^{26}$

De acuerdo a lo anterior es posible encontrar entonces una mixtura en la que la clase baja intente acercarse a un orden proyectado por la elite. En este proceso de blanqueamiento, la clase baja dice lo que cree que la clase alta va a decir. El carácter de moral entrepreneur se traslada entonces - sólo en un plano discursivo- a la clase baja porque sus miembros han percibido en la construcción de la identidad chilena la función paternalista de la clase alta. $R$ especto a la televisión se puede señalar que la clase baja puede entonces, en un intento imitativo, emitir juicios que señalen la necesidad de restringir la transmisión de contenidos o bien señalar que ésta es negativa para ciertos valores culturales promovidos por la elite. Esto último implicaría entonces sugerir que la función de emprendimiento moral se ejerce en la emisión de juicios.

N o obstante lo anterior, son ciertas conductas en el ámbito privado, las cua les están asociadas a la analogía del color de la piel, las que finalmente señalan la existencia de una conciencia dual. En esta esfera la utilización y creación de un lenguaje por parte del dominado (i.e. créole) resume elocuentemente dicha contradicción. R especto de la televisión, sería plausible entonces encontrar conductas que contradigan el blanqueamiento de la clase baja, puesto que en dicha esfera las exigencias de aparecer como el dominante son reducidas

${ }^{26} \mathrm{C}$ iertamente hay muchas diferencias entre los contextos social es que dicho autor revisa con respecto a la colonización vivida por $C$ hile, sin embargo, aquí interesa revisar en términos sociológicosla aparición de esta dualidad, la cual se manifiesta culturalmente, en aquellos grupos que han sido dominados, o que al menos no son parte de la elite colonizadora. Es decir, aun cuando éste sea explícitamente una teoría sobre el racismo como forma de dominación, es de vital importancia señalar cómo ciertos valores finalmente se terminan invirtiendo. 
La figura 2 muestra cómo estaría desplegándose la función de moral entrepreneur en consideración con los conceptos tradicionalismo ideológico y conciencia dual, tomando eso sí las dimensiones esfera pública y esfera privada.

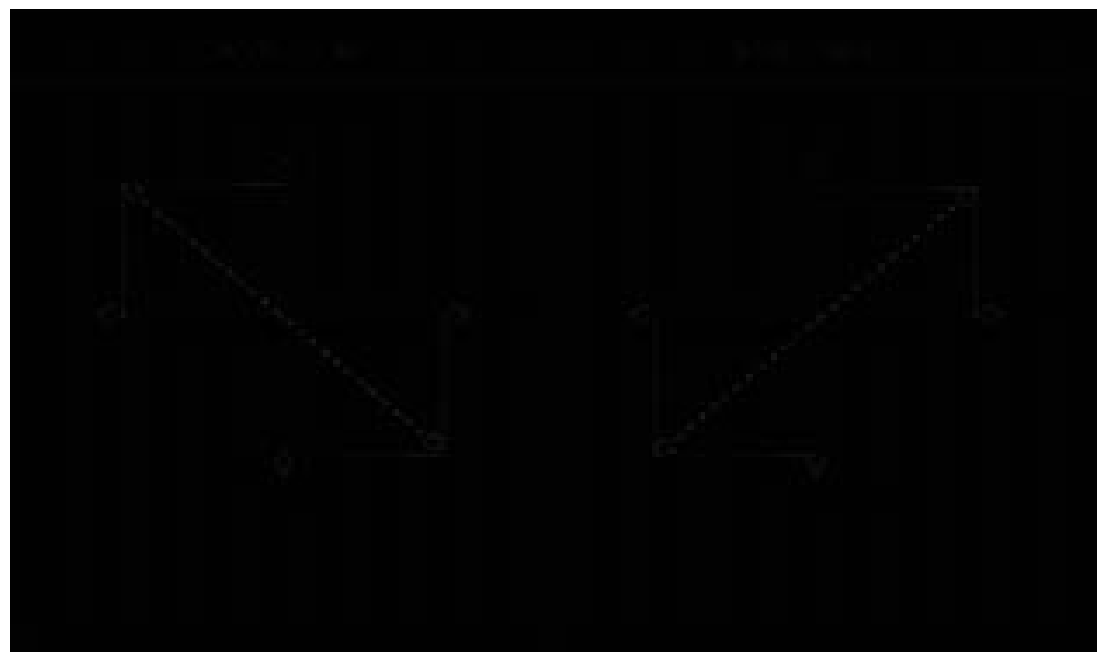

Figura 2. Caracterización teórica alternativa del funcionamiento del emprendimiento moral con relación a las clases sociales para la esfera pública y esfera privada.

\section{Despliegue analítico del emprendimiento moral en torno a la televisión}

G racias a la distinción analítica: i) esfera pública = televisión; ii) efectos negativos de la televisión; iii) control sobre los hijos en la esfera privada, es posible realizar una operacionalización sobre cómo se despliega la función de emprendimiento moral en torno a la televisión. Para lo anterior se revisarálasimplicancias teóricas de cada una de estas dimensiones.

\section{1) ESFERA PÚBLICA = TELEVISIÓN}

D ebido a cambios dramáticos en el área tecnológica y en los procesos demográficos del siglo anterior, el concepto de esfera pública puede ser muy elusivo. N o obstante, hay en la propia definición habermasiana, de la esfera pública del siglo XIX rastros que permiten señalar que la televisión es = a la esfera pública (H abermas, 1989). Lo anterior porque parte esencial de ésta son instituciones como parlamentos, salones literarios, asambleas públicas, pubs, cafés, salones, pero también medios masivos tales como diarios y revistas. Es precisamente la aparición de los medios masivos la que sugiere a la televisión como una institución constituyente de la esfera pública. C abe precisar sin embargo, que el actual funcionamiento de la televisión, dado que existe una intervención explícita por parte del Estado como una comercialización de la prensa hace problemática asumir esta igualdad. Esto porque no necesariamente queda garantizada la transmisión libre de los discursos, a través de los contenidos que emite la televisión. 
Sin embargo, más allá de que la esfera pública pueda estar colonizada por ciertos intereses, en este trabajo lo que importa es asumir el concepto ideal de esfera pública propuesto por Habermas. Ésta es entendida como una red de actores privados que reunidos como público pueden articular las necesidades de la sociedad con el estado (H abermas, 1989: 176). Es decir como red en la cual los actores pueden compartir información y puntos de vista (H abermas, 1999: 360). Bajo este criterio la televisión puede ser entonces entendida como una institución que forma parte de la esfera pública. Lo anterior además se fundamenta en que la televisión en sí es potencialmente un medio por donde pueden circular en forma libre o sin coerción los distintos puntos de vista de todos los actores sociales, pero además porque en C hile ésta ha jugado un rol muy importante en la ampliación del proceso democrático (M arín y Cordero, 2005). En consecuencia y dado que la televisión potencialmente permite que todos los puntos de vistas sean comunicados, la función de emprendimiento moral se manifestará en pregonar mayores grados de censura para ésta.

\section{II) Efectos negativos de LA teleVisión}

D iversos estudios han reconocido que la televisión puede tener ciertos efectos negativoso nocivos en sus espectadores(Josephson, 1997; Joy, K imball y Zabrack, 1986). Por otra parte hay otros que señalan que a lo sumo los efectos de ésta estarían mediados por otros factores (H all, N eitz y Battani, 2003; M cN eely, 1997). N o obstante, resulta difícil señalar claramente hacia cuál de estos polos se puede asociar el ethos del emprendimiento moral. Brunner y $C$ atalán señalan que son los grupos conservadores los que asocian el aprendizaje de conductas impropias a la televisión. Esto autores reconocen que ciertos grupos minoritarios pero poderosos pugnan «ntensamente por el control de la televisión, [ya que] el público consume desaprensivamente 'basura'» (B runner y C atalán, 1995: 101-2). Esto es, el emprendimiento moral se manifiesta también en la creencia de que los auditores sean sujetos completamente vulnerables a los contenidos y no sepan seleccionar de éstos aquello que es apropiado.

En esta línea argumentativa también se encuentra el trabajo desarrollado por Bourdieu (1998), quien ubica su análisis en los contenidos que la televisión transmite más que en los efectos que operan en las conductas de los televidentes. Es decir, este autor asume que la televisión tiene efectos, pero éstos dependen de los contenidos que son emitidos. En ese entendido la televisión efectivamente, señala este autor, ha producido una banalización en los auditores, y hace que éstos dejen de lado cualquier effuerzo por reflexionar con mayor profundidad, tal como lo demanda un orden social democrático. En este entendido Bourdieu, al igual que $\mathrm{H}$ abermas, implícitamente entiende que la televisión puede ocupar un rol libertario, si es que a través de ésta la información no está ajustada exclusivamente a grupos de interés dominantes. Para los fines de este trabajo, Bourdieu representa un perfecto emprendedor moral porque puja desde una mirada paternalista por la liberación de sectores «dormidos» por los efectos de la televisión.

En consecuencia la función de emprendimiento moral se puede ejercer a 
través de la creencia de que la televisión afecta negativamente (sin considerar factores intermedios) los valores y conductas de las personas. Esto es, los telespectadores son entendidos como meros receptores de información y con nula capacidad de discernimiento respecto de lo que la televisión transmite.

\section{III) CONTROL EN LA ESFER A PRIVADA}

\section{SOBRE EL CONSUMO DE TELEVISIÓN DE LOS MENORES}

Establecer una distinción entre esfera privada y esfera pública, como sostiene Goffman, es bastante problemático ya que la construcción del self dependerá entre otros de los contextos culturales, organizacionales, familiares por donde éste va transitando (Goffman, 1959: 252-3). En cada uno de estos contextos, señala G offman, la dicotomía privado/ púbico siempre se produce. Por ejemplo, dentro de una «total institution» el self está tan constreñido por losfuncionarios, que el baño es uno de los pocos lugares privados al cual el interno tiene acceso (G offman, 1961: 119). Considerando lo anterior, y para efectos de este trabajo, el control de la televisión que hacen los padres en el hogar representa la esfera privada. En contraposición a ésta y como se vio antes, la representación de la esfera pública está dada por la televisión.

$R$ especto a cómo se ejerce la función de emprendimiento moral en esta esfera, se puede señalar que será aquella representada por los adultos que realicen un control directo e indirecto sobre lo que los menores del hogar ven. Lo anterior porque esto constituye una acción en la cual está sintetizada tanto la construcción de una norma dentro de la casa, como también la propia observación de ésta, y son precisamente los adultos los que la llevan a cabo.

\section{M etodología}

\section{D atos}

Los datos utilizados en este estudio provienen de la Encuesta $\mathrm{N}$ acional de Televisión realizada por el Consejo N acional de Televisión durante el año 2005. D icha encuesta entrevistó en forma al eatoria a 2770 personas utilizando entrevistas cara a cara. Para efecto de revisar las tesis aquí propuestas la muestra se ha dividido en dos: (i) se considera un grupo donde no se diferencia si hay menores de trece años en el hogar entrevistado $(n=2615)$, y (ii) un grupo cuyos hogares tenían al menos un menor de trece años viviendo en él, y que respondieron a cada una de las preguntas analizadas. Este segundo grupo es el utilizado para evaluar los aspectos asociados a la esfera privada $(n=1257)$.

\section{Dimensiones}

D ado que hay tres tesis propuestas, se seleccionaron las siguientes tres dimensiones para analizar el ejercicio de la función de emprendimiento moral (ver tabla 2 para sus estadísticos descriptivos): 
(i) Dimensión: Esfera pública = televisión. Lasalternativas representan una gradación de censura en torno a la televisión:

1. «nexistencia de ley que regule la transmisión de programas;;

2. « ebe haber horario definido para adultos, donde los canales pueden transmitir lo que quieran $»_{i}$

3. « ebiera haber un horario para la programación de adultos, y en ese horario se deben regular sólo algunos contenidos extremos); y

4. «D ebiera haber una estricta regulación, tanto de horarios como de contenidoss.

(ii) Dimensión:Efectosnegativos de la televisión. ${ }^{37}$ Las al ternativas representan distintas creencias sobre los efectos de la televisión:

1. «a televisión hace que losjóvenestengan conductassexualesinapropiadas»;

2. «a televisión estimula el consumismo en los niños, porque quieren comprar todo lo que sale en la televisión»;

3. «a televisión incentiva la violencia en las personas); y

4. «a televisión hace que a los niños les vaya mal en el colegio».

(iii) Dimensión:C ontrol en la effera privada sobre el consumo de televisión de los menores. Las alternativas representan la supervisión directa o indirecta que los adultos del hogar ejercen sobre los niños:

1. «H ay un adulto supervisando directamente lo que un niño menor de 13 años ve en televisión»;

2. «Q ué cantidad de tiempo los niños menores de 13 años ven televisión (M ucha, A decuada, Poca)».

\section{Clase social}

D ebido a que la diferencia cultural entre clases sociales respecto a la televisión es lo que guía este artículo, es pertinente definir metodológicamente que se entiende por clase social en este trabajo.

Para definir clase social se optará por la propuesta desar rollada por Bourdieu (2000). D e acuerdo a este autor la adquisición o propiedad de capitales económico, cultural (informacional), social y simbólico puede sintetizar lo que las personas tienen y por tanto ubicarlas en una clase social. D os elementos centrales para esta clasificación que aquí se utiliza son capital económico y cultural. $R$ especto del primero se utilizará los bienes económicos que las per sonas declaran tener, y con relación al segundo, su nivel educacional. Evidentemente, esto

${ }^{37}$ Para medir esta dimensión se construyó un índice con cuatro indicadores. Se obtuvo un á de $C$ ronbach de 0,646 para la primera submuestra, y de 0,635 para la segunda submuestra. 
Tabla 1. Estadísticas descriptivas de las dimensiones

\begin{tabular}{|c|c|c|c|c|c|c|c|}
\hline \multirow{2}{*}{ Dimensiones } & \multirow{2}{*}{ Alternativas } & \multicolumn{3}{|c|}{$\begin{array}{c}\text { Submuestra } \\
\text { Con hijos y sin hijos }\end{array}$} & \multicolumn{3}{|c|}{$\begin{array}{l}\text { Submuestra } \\
\text { Sólo con hijos }\end{array}$} \\
\hline & & $\%$ & Media & $\begin{array}{l}\text { Desviación } \\
\text { Estándar }\end{array}$ & $\%$ & Media & $\begin{array}{l}\text { Desviación } \\
\text { Estándar }\end{array}$ \\
\hline \multirow{4}{*}{$\begin{array}{l}\text { (i) Esfera pública = } \\
\text { televisión }\end{array}$} & (1) & 5,8 & & & 4,7 & & \\
\hline & (2) & 40,2 & & & 39,5 & & \\
\hline & (3) & 29,0 & & & 29,2 & & \\
\hline & (4) & 25,0 & & & 26,5 & & \\
\hline $\begin{array}{l}\text { (ii) Efectos negativos de } \\
\text { la televisión }\end{array}$ & & & 2,37 & 0,67 & & 2,28 & 0,67 \\
\hline \multirow{2}{*}{$\begin{array}{l}\text { (iii) Esfera privada } \\
\text { (Supervisión directa) }\end{array}$} & $\begin{array}{l}\text { Adulto } \\
\text { controla }\end{array}$ & - & & & 86,9 & & \\
\hline & $\begin{array}{c}\text { Ningún } \\
\text { adulto controla }\end{array}$ & - & & & 13,1 & & \\
\hline \multirow{3}{*}{$\begin{array}{l}\text { (iii) Esfera privada } \\
\text { (Supervisión indirecta) }\end{array}$} & Mucha & - & & & 34,9 & & \\
\hline & Adecuada & - & & & 53,4 & & \\
\hline & Poca & - & & & 11,7 & & \\
\hline
\end{tabular}

no es otra cosa que un clasificador socioeconómico, o más bien edu-económico porque de social tiene poco (R ytina, 2000). Sin embargo, la categorización derivada de la unión de estas dos características permite clasificar a las personas en clases. De acuerdo a lo anterior y para evitar confusiones o desilusiones metodológicas, dos elementos deben ser mencionados. Primero, y en coherencia a 0 ssow ski (1963), clase tiene aquí un sentido objetivo en tanto ubica a las personas en un esquema de gradación de distribución desigual de recursos. ${ }^{41}$ Segundo, clase sintetiza la adquisición de recursos asociados a bienes económicos junto a los elementos asociados al prestigio (honor) social. ${ }^{42}$ En síntesis, de acuerdo a la metodología utilizada para medir grupos socioeconómicos, clase social es un constructo en el cual se sintetizan niveles de educación con la adquisición de bienes de materiales. Lo anterior da como resultado cinco clases (alta, media-alta, media, media-baja y baja).

\section{Variables control}

D e forma de aislar la influencia de otras variables, cuyos efectos fueran significativos en las posiciones culturales de los individuos, fueron introducidas religión,

${ }^{41}$ Bajo este precepto queda fuera de esta discusión toda la tradición marxista en tanto ésta busca responder a cuáles son las tranforormaciones necesarias que eliminarían la explotación y la opresión económica dentro de las sociedades capital istas (ver W right, 1997: 1-39).

${ }^{42}$ Esto último tiene sin duda un fuerte eco teórico en Weber, no obstante y en estricto rigor, el concepto de clase que este autor trabaja está dado exclusivamente por la posición que la persona tiene en el mercado, siendo el status el que le permite referirse a los recursos asociados al honor y al prestigio, los cuales no podrían transarse necesariamente en forma inmediata (Weber, 1944). 
sexo, edad y número de hijos como controles. Especial énfasis hay que poner sobre religión (M orandé, 1984; Larraín, 2001: 235-41; O ssa, 1996), ya que para el caso chileno éste es constitutivo de la identidad nacional y puede causar distorsiones en el análisis de los datos. C on respecto a sexo diversos estudios señalan las diferencias culturales entre hombres y mujeres, el cual en consecuencia debe también ser considerado (M ontecino, 1996; O lavarría, 2000;Valdés y Valdés, 2005; Varios autores, 2005). R especto a la edad, también hay diversas investigaciones que señalan las diferencias entre jóvenes, adultos y adultos ma yores (Duarte, 2002; A ranibar, 2001). Finalmente con respecto al número de hijos, se estimó pertinente introducir dicho dato debido a que, dicha característica podía dar cuenta de situaciones divergentes al interior de los hogares. Al respecto no se encontró datos que pudieran rechazar o aprobar dicha afirmación.

Tabla 2. Estadísticas descriptivas de Variables I ndependientes

\begin{tabular}{|c|c|c|c|c|c|c|c|}
\hline \multirow{2}{*}{\multicolumn{2}{|c|}{ Variables independientes }} & \multicolumn{3}{|c|}{$\begin{array}{c}\text { Submuestra } \\
\text { Con hijos y sin hijos }\end{array}$} & \multicolumn{3}{|c|}{$\begin{array}{l}\text { Submuestra } \\
\text { Sólo con hijos }\end{array}$} \\
\hline & & & & Desviación & & & Desviación \\
\hline \multirow{5}{*}{ Clase social } & Alta & 10.9 & & & 8.4 & & \\
\hline & Media Alta & 18.9 & & & 17.5 & & \\
\hline & Media & 25.6 & & & 23.9 & & \\
\hline & Media Baja & 34.6 & & & 38.7 & & \\
\hline & Baja & 10.0 & & & 11.4 & & \\
\hline \multirow{6}{*}{ Religión (Control) } & Católica Ortodoxa & 43.3 & & & 40.0 & & \\
\hline & Católica Heterodoxa & 22.0 & & & 22.1 & & \\
\hline & Evangélica Ortodoxa & 10.5 & & & 13.7 & & \\
\hline & $\begin{array}{l}\text { Evangélica } \\
\text { Heterodoxa }\end{array}$ & 4.1 & & & 6.0 & & \\
\hline & Otras religiones & 5.1 & & & 4.9 & & \\
\hline & Secular & 14.9 & & & 13.2 & & \\
\hline \multirow{2}{*}{ Sexo (Control) } & Hombre & 48.5 & & & 41.0 & & \\
\hline & Mujer & 51.5 & & & 59.0 & & \\
\hline Edad (Control) & & & 40.24 & 16.58 & & 35.93 & 12.83 \\
\hline $\begin{array}{l}\text { Número de hijos en la } \\
\text { casa (1-11) (Control) }\end{array}$ & & - & & & & 2.43 & 1.41 \\
\hline $\mathrm{N}$ & & 2615 & & & 1257 & & \\
\hline
\end{tabular}

\section{R esultados y análisis}

Para determinar las tesis aquí propuestas se aplicaron para las dos submuestras tres técnicas estadísticas diferenciadas. Para (i) esfera pública = televisión, se utilizó regresión logística multinomial. Esta permite establecer la relación entre clases sociales y los juicios (1), (2), (3), y (4), esto es conocer qué clase se inclina más 
por uno o por otro control a la televisión, cuando clase alta es la referencia a tener en cuenta. Para (ii) Efectosnegativos de la televisión se creó un índice con valores continuos y por tanto se utilizó regresión lineal. Finalmente, para (iii) Control en la esfera privadasobreel consumo de televisión de loshijos, se aplicó para(1) supervisión directa, regresión logística dicotómica ya que esta variable tenía sólo dos valores (ausencia o presencia de un adulto supervisando lo que el menor ve); y para (2) supervisión indirecta, se utilizó nuevamente regresión logística multinomial, porque la variable tenía tres categorías. Los resultados son analizados a partir de cada una de las tesis.

\section{Análisis de (i) Dimensión: Esfera pública = televisión}

Al analizar los resultados obtenidos en la regresión logística multinominal para la primera sub-muestra (tabla 3), ${ }^{46}$ siendo la categoría de referencia de la variable dependiente (4) Estricta R egulación y habiendo introducidos los controles que aparecen en la literatura (los cuales la mayoría de ellos resultaron ser estadísticamente significativos), se observa que todas las clases despliegan una mayor función de emprendimiento moral que la clase alta, puesto que los signos de los valores para cada uno de los parámetros es negativo.

D e acuerdo a lo anterior cada una de las clases tiene mayores probabilidades de optar por la categoría (4) Estricta R egulación que por cualquiera de las otras 3 categorías, las cuales operacionalizan menores grados de censura. Es preciso reconocer, sin embargo, que la clase al ta con la clase baja sólo tienen diferencias estadísticas cuando son comparadas las categorías (3) y (4). R especto de los parámetros obtenidos para las clases media alta, media y media-baja, éstos son estadísticamente significativos al compararse con la clase alta.

Para poder tener un mejor entendimiento de lo que la tabla 3 informa, se puede comparar el caso de una persona asociada a la clase baja y determinar las chances de ésta por optar por una categoría que represente algún grado de censura menor en comparación a una persona de clase alta. Por ejemplo (ver en tabla 3 cuadro sombreado), una persona de clase baja tiene (e.558) 1.75 veces más chances, que una persona de clase alta, de optar por un mecanismo de control (3) en el cual se sugiere tener horario para la programación de adultos, y en ese horario se deben regular sólo algunos contenidos extremos, que de (4) aprobar la estricta regulación, tanto de horarios como de contenidos.

Si la clase al ta está mostrando una menor función de emprendimiento moral respecto de la censura que debiese ser impuesta a la televisión, el concepto de

${ }^{46} \mathrm{El}$ modelo multinomial puede ser presentado de la siguiente manera.

$$
\log \left(\frac{P_{k}}{P_{(4)}}\right)=X^{\prime} \beta, \quad k=(1),(2),(3)
$$

D onde $P_{(4)}$ es la probabilidad que una persona opte por una censura del tipo «4» (Estricta $\mathrm{R}$ egulación), $\mathrm{P}_{k}$ es la probabilidad que una persona responda a una categoría $k, X$ es el vector de los covariados, y $ß$ el vector de los parámetros a ser estimado. 
Tabla 3. Modelo de regresión para

«(i) Dimensión: Esfera pública = televisión» (continuación)

\begin{tabular}{|c|c|c|}
\hline \multicolumn{2}{|c|}{2 VERSUS 4} & \\
\hline \multirow{5}{*}{ Clase social } & Media Alta & $-.669 * *(.512)$ \\
\hline & Media & $-.609 * *(.544)$ \\
\hline & Media Baja & $-.392 * \quad(.675)$ \\
\hline & Baja & $-.090 \quad(.914)$ \\
\hline & Alta (referencia) & \\
\hline \multirow{6}{*}{ Religión (Control) } & Católica Ortodoxa & $-.137(.872)$ \\
\hline & Católica Heterodoxa & $.197(1.218)$ \\
\hline & Evangélica Ortodoxa & $-.464 *(.629)$ \\
\hline & Evangélica Heterodoxa & $-.017(.983)$ \\
\hline & Otras religiones & $-.514 *(.598)$ \\
\hline & Secular (referencia) & \\
\hline Sexo (Control) & Mujer & $.572 * * *(1.772)$ \\
\hline Edad (Control) & & $-.022 * * *(.978)$ \\
\hline \multicolumn{3}{|c|}{ Número de hijos en la casa (1-11) (Control) } \\
\hline Constante & & $1.687 * * *$ \\
\hline \multicolumn{2}{|c|}{3 VERSUS 4} & \\
\hline \multirow{5}{*}{ Clase social } & Media Alta & $-.647 * *(.524)$ \\
\hline & Media & $-.727 * *(.483)$ \\
\hline & Media Baja & $-.728 * * *(.483)$ \\
\hline & Baja & $-.583^{*}(.558)$ \\
\hline & Alta (referencia) & \\
\hline \multirow{6}{*}{ Religión (Control) } & Católica Ortodoxa & $-.134(.875)$ \\
\hline & Católica Heterodoxa & $.433^{*}(1.542)$ \\
\hline & Evangélica Ortodoxa & $-.246(.782)$ \\
\hline & Evangélica Heterodoxa & $.054(1.056)$ \\
\hline & Otras religiones & $-.359(.698)$ \\
\hline & Secular (referencia) & \\
\hline Sexo (Control) & Mujer & $.364 * *(1.439)$ \\
\hline Edad (Control) & & $-.015 * * *(.986)$ \\
\hline \multicolumn{3}{|c|}{ Número de hijos en la casa (1-11) (Control) } \\
\hline Constante & & $1.259 * * *$ \\
\hline Pseudo $R^{2}$ & & .077 \\
\hline N & & 2603 \\
\hline
\end{tabular}




\section{Análisis de (ii) Efectos negativos de la televisión}

Al analizar esta segunda dimensión, la cual permite distinguir si las clases manifiestan diferenciadamente sus juicios respecto a los efectos negativos de la televisión, se puede señalar que los resultados son similares a la dimensión analizada anteriormente. U na vez introducidos los controles, se puede observar en la tabla 4, que la única clase que no tiene diferencias estadísticas con la clase alta (teniendo como referencia el índice construido para sintetizar los diferentes enunciados que operacionalizan efectos negativos de la televisión) es la clase media-alta.

En otras palabras la potencial función de emprendimiento moral puede ser actualizada por las clases baja, media y media-baja, por cuanto ellas creerían que las acciones, actitudes y valores de las personas son vulnerables a los contenidos transmitidos por la televisión, y no a otros factores intermedios. Contrario a lo anterior las clases alta y media-alta, al estar más cercana a la materialización del tradicionalismo ideológico, entenderían que son otros factores los que pueden producir efectos más nocivos en sus valores y por tanto podrían introducir mecanismos de control más efectivos en la esfera privada.

Tabla 4. Modelo de regresión «Efectos negativos de la televisión»

\begin{tabular}{|c|c|c|}
\hline \multicolumn{2}{|c|}{} & $\begin{array}{c}\text { Sub muestra } \\
\text { Con hijos y sin hijos }\end{array}$ \\
\hline \multirow{4}{*}{ Clase social } & Media Alta (C2) & $.059(.034)$ \\
\cline { 2 - 3 } & Media (C3) & $180 * * *(.117)$ \\
\cline { 2 - 3 } & Media Baja (D) & $.137^{* *}(.097)$ \\
\cline { 2 - 3 } & Baja (E) & $.126^{*}(.056)$ \\
\hline \multirow{3}{*}{ Religión (Control) } & Clta (ABC1) (referencia) & $.008(.006)$ \\
\cline { 2 - 3 } & Católica Ortodoxa & $-.047(-.029)$ \\
\cline { 2 - 3 } & Cválica Heterodoxa & $.126 * \mathrm{~L}(.058)$ \\
\cline { 2 - 3 } & Evangélica Heterodoxa & $.035(.010)$ \\
\hline Sexo (Control) & Otras religiones & $.169 * \mathrm{~L}(.056)$ \\
\hline Edad (Control) & Secular (referencia) & $-.087 * *(-.065)$ \\
\hline Número de hijos en la casa & Mujer & $-.007 * * *(-.168)$ \\
\hline (Control) & & \\
\hline Constante & & $2.121^{*} * * *$ \\
\hline $\mathrm{R}^{2}$ & & .053 \\
\hline $\mathrm{N}$ & & 2603 \\
\hline
\end{tabular}

Los betas estandarizados están entre paréntesis

$* p<.05 \quad * * p<.01 \quad * * * p<.001$

L denota un coeficiente del grupo Católico ortodoxo que es significativamente menor que otro grupo religioso.

$\iota_{p}<.05 \quad \iota_{p}<.01{ }^{\omega_{p}} \iota_{p}<.001$ 
Finalmente, los resultadossugieren también que la función de emprendimiento moral para esta dimensión estaría más asociada a las clases bajas, dado que la creencia sobre la negatividad de la televisión habría sido percibido por partes de miembros de este grupo como parte constituyente de un discurso paternalista de las clases altas. En tanto los miembros de las clases bajas dicen lo que habrían dicho las clases altas, la manifestación de lo anterior permite que el proceso de blanqueamiento se pueda producir.

\section{Análisis de (iii) Dimensión: Control en la esfera privada sobre el consumo de televisión de los menores}

Para determinar el tipo de control que los distintos miembros de las clases ejercen al interior de sus hogares, se optó por subdividir el tipo de control ejercido sobre el consumo de televisión de los menores entre supervisión directa y supervisión indirecta.

\section{Supervisión directa}

C omo se puede apreciar en la tabla 5, los datos indican que los menores de las clases bajas tienen mayores probabilidades de ver televisión sin la presencia de un adulto, que los menores de cualquier otra clase (con la excepción de la clase media alta). Al tomar como ejemplo la comparación de un menor de clase alta con uno de clase baja (ver cuadro sombreado con gris), éste último tiene $\left(e^{2.239}\right)$ 9.48 veces menos chances de ser controlado por un adulto.

C abe señalar que este resultado indica que la clase alta funciona de acuerdo al concepto de tradicionalismo ideológico acá revisado. Esto porque dicho grupo, deja de lado el control sobre ciertos procesos asociados a la esfera pública, y sí ejerce estrategias de mayor control en la esfera privada. Lo anterior implica que en este ámbito dicho grupo sí despliega una función de emprendimiento moral pues mantiene a un adulto supervisando cuando un menor ve televisión.

Con respecto a la clase baja, su función a diferencia de las analizadas en las otras dos dimensiones, no tiene una forma de emprendimiento moral. Esto sí estaría más asociado a lo que plantea Fanon y tangencialmente Salazar, porque en la esfera privada desarrollan patrones culturales que se distancia de las conductas de elite. En consecuencia, en esta esfera el proceso de blanqueamiento se ve enfrentado a condiciones objetivas que dificultan su desarrollo.

Esto último merece al menos señalar algo sobre los mecanismos sociales ${ }^{47}$ que estarían facilitando o inhibiendo la función de emprendimiento moral al interior de la esfera privada en estas clases. Considerando la división en términos salariales de estas clases, se podría sugerir que dado el ingreso de las clases altas éstas tienen mayores posibilidades de supervisar a un menor del hogar por dos situaciones que no necesariamente son excluyentes: primero, existe un adulto perteneciente a la familia que se puede encargar de tareas domésticas. Segundo, hay un adulto contratado (the so-called asesoras del hogar) el cual, entre otras fun-

${ }^{47}$ Sobre mecanismos sociales verVan den Berg (1998). 
ciones formales al interior del hogar, tiene la supervisión directa de las actividades que realizan los menores.

Con respecto a la clase baja, se puede señalar que la mayor ausencia de un adulto en el hogar se da también por las dos razones esgrimidas respecto a la clase alta, en un orden contrario, y también una tercera. Primero, es necesaria la inclusión de todos los adultos del hogar al mercado laboral para mejorar el ingreso; segundo, el ingreso que estas familias recibe no alcanza para contratar una persona que trabaje al interior del hogar ejerciendo funciones de supervisión hacia los menores, y finalmente, y al menos para el caso de la región metropolitana, la ausencia en el hogar se potencia cuando se toma también en consideración los tiempos en los traslados de estas personas, cuando ellas utilizan el transporte público para dirigirse al trabajo o bien para volver al hogar.

Tabla 5. Modelos de regresión logística dicotómica «(iii) Dimensión: Control en la esfera privada sobre el consumo de televisión de los menores (Supervisión directa)»

\begin{tabular}{|c|c|c|}
\hline \multicolumn{2}{|c|}{} & $\begin{array}{c}\text { Submuestra } \\
\text { Sólo con hijos }\end{array}$ \\
\hline \multirow{4}{*}{ Clase social } & Alta & $.811^{*}(2.250)$ \\
\cline { 2 - 3 } & Media Alta & $.443(1.558)$ \\
\cline { 2 - 3 } & Media & $.548 *(1.730)$ \\
\cline { 2 - 3 } & Media Baja & $.539 *(1.714)$ \\
\hline \multirow{3}{*}{ Religión (Control) } & Baja (referencia) & \\
\cline { 2 - 3 } & Católica Ortodoxa & $-.219(.803)$ \\
\cline { 2 - 3 } & Católica Heterodoxa & $-.464(.629)$ \\
\cline { 2 - 3 } & Evangélica Ortodoxa & $-.014(.986)$ \\
\cline { 2 - 3 } & Evangélica Heterodoxa & $-.330(.719)$ \\
\cline { 2 - 3 } & Otras religiones & $.802(2.230)$ \\
\hline Sexo (Control) & Secular (referencia) & $-.288(.749)$ \\
\hline Edad (Control) & Mujer & $-.015 *(.985)$ \\
\hline Número de hijos en la casa (1-11) & & $.097(1.102)$ \\
\hline (Control) & & $-2.989 * *(.050)$ \\
\hline Constante & & .038 \\
\hline Pseudo R & & 1227 \\
\hline $\mathrm{N}$ & & \\
\hline
\end{tabular}

Los exponentes de los betas están entre paréntesis

$* p<.05 \quad * * p<.01 \quad * * * p<.001$

\section{Supervisión indirecta}

Con relación a la supervisión indirecta los datos de la tabla 6 (específicamente por el valor negativo de los parámetros asociado a cada valor de la variable clase social) indican que en general los menores de las clases bajas ven más televisión 
Tabla 6. Modelos de regresión logística dicotómica «(iii) Dimensión: Control en la esfera privada sobre el consumo de televisión de los menores (Supervisión indirecta)»

\begin{tabular}{|c|c|c|}
\hline & & $\begin{array}{l}\text { Submuestra } \\
\text { Sólo con hijos }\end{array}$ \\
\hline ADECUADA VE & MUCHA & \\
\hline & Alta & $-.183(.833)$ \\
\hline & Media Alta & $-.469 *(.626)$ \\
\hline Clase social & Media & $-.258(.773)$ \\
\hline & Media Baja & $-.239(.788)$ \\
\hline & Baja (referencia) & \\
\hline & Católica Ortodoxa & $.032(1.032)$ \\
\hline & Católica Heterodoxa & $.316(1.372)$ \\
\hline Doliaión (Control) & Evangélica Ortodoxa & $.106(1.111)$ \\
\hline 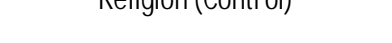 & Evangélica Heterodoxa & $-.088(.915)$ \\
\hline & Otras religiones & $-.124(.883)$ \\
\hline & Secular (referencia) & \\
\hline Sexo (Control) & Mujer & $.1421(1.153)$ \\
\hline Edad (Control) & & $-.003(.997)$ \\
\hline $\begin{array}{l}\text { Número de hijos en la casa (1-11) } \\
\text { (Control) }\end{array}$ & & $-.084(.919)$ \\
\hline Constante & & $.855 * *$ \\
\hline POCA VERS & UCHA & \\
\hline & Alta & $.011(1.011)$ \\
\hline & Media Alta (C2) & $-.505(.603)$ \\
\hline Clase social & Media (C3) & $.504(1.655)$ \\
\hline & Media Baja (D) & $.118(1.125)$ \\
\hline & Baja (E) (referencia) & \\
\hline & Católica Ortodoxa & $-.352(.703)$ \\
\hline & Católica Heterodoxa & $-.106(.899)$ \\
\hline Doligión (Control) & Evangélica Ortodoxa & $.032(1.033)$ \\
\hline 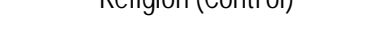 & Evangélica Heterodoxa & $.005(1.005)$ \\
\hline & Otras religiones & $-.411(.663)$ \\
\hline & Secular (referencia) & \\
\hline Sexo (Control) & Mujer & $-.174(.840)$ \\
\hline Edad (Control) & & $.009(1.009)$ \\
\hline $\begin{array}{l}\text { Número de hijos en la casa (1-11) } \\
\text { (Control) }\end{array}$ & & $.010(1.010)$ \\
\hline Constante & & $-1.303 * *$ \\
\hline Pseudo $R^{2}$ & & .032 \\
\hline $\mathrm{N}$ & & 1217 \\
\hline
\end{tabular}


que aquellos menores de las otras clases sociales. N o obstante lo anterior, sólo la clase baja con la clase media-alta, cuando son comparadas las categorías «mucha» y «adecuada» cantidad de televisión, tienen diferencias estadísticamente significantes. $R$ especto al resultado obtenido se puede señalar que un menor de clase media alta tiene $\left(e^{.626}\right)$ 1,87 veces más chances de ver una cantidad «adecuada» de televisión que uno de clase baja, cuando es tomado como referencia «mucha» cantidad de televisión (ver en tabla 6 cuadro sombreado con gris).

$D$ adas las significancias estadísticas obtenidas, es preciso ser cautel oso respecto a proponer qué clase social estaría ejerciendo una supervisión indirecta. N o obstante, y cómo se mencionó sobre los signos negativos de los parámetros obtenidos, se puede inferir que la función de emprendimiento moral en la esfera privada, al menos no está siendo ejecutada por la clase baja. Esto último estaría relacionado parcialmente con los datos analizados en la tabla 5, porque la clase baja estaría ejerciendo menos control sobre la cantidad de televisión que ven los menores de sus hogares. Evidentemente consumir más televisión no necesariamente significa per se que haya un menor control en la esfera privada, sin embargo tras lo analizado en las dimensiones (i) y (ii), esto es difícil de sostener, puesto que la clase baja en general declara que la televisión requiere mayores controles y produce efectos negativos en las personas.

Al igual que en el análisis realizado para determinar la supervisión directa, los resultados para este tipo de supervisión podrían también ser explicados por el funcionamiento específico de mecanismos sociales asociados a las diferencias en losingresos, o como lo sostiene Lechner «el tradicionalismo [ideológico] representa una estrategia viable para los grupos de nivel socioeconómico alto. Pero no es una opción para la mayoría de la población que sufre la sobrecarga de la familia tradicional» (1999: 3). Esto último se puede complementar con la metáfora de Fanon, la esfera privada inevitablemente se mantiene negra para la clase baja y blanca para la clase alta. Las máscaras se pueden invertir pero el color de la piel queda.

\section{Un nuevo emprendedor moral en función y forma}

Los datos analizados permiten señalar que la función del emprendimiento moral está presente en la sociedad chilena, pero ésta se actualiza según ámbito de acción y clase social. Esto es, la ejecución de esta función se produce diferenciadamente para las esferas privadas y públicas. De acuerdo a lo anterior, en primer término se puede observar que la función de emprendimiento moral desplegada en el ámbito privado es hecho mayormente por las clases altas, y está dirigido a menores que habitan dichos hogares. En segundo término, la función de emprendimiento moral dirigida hacia la esfera pública enraizada en las clases bajas, sólo podría considerarse latente. Lo anterior porque los valores de estas clases no han colonizado los contenidos transmitidos por la televisión. C on rela ción a la ausencia de colonización de estos valores, se puede sugerir a modo de hipótesis lo siguiente: dicho proceso no ha ocurrido porque miembros de las clases bajas carecen de niveles de coordinación y esto redunda en bajos niveles de presión. U na segunda alternativa (la cual no es necesariamente excluyente) 
sería que la televisión está clausurada a recibir información que atente contra un funcionamiento asociado a principios económicos.

A continuación se presenta en figura 3 el funcionamiento de emprendimiento moral para el caso de la televisión al interior de la sociedad chilena.

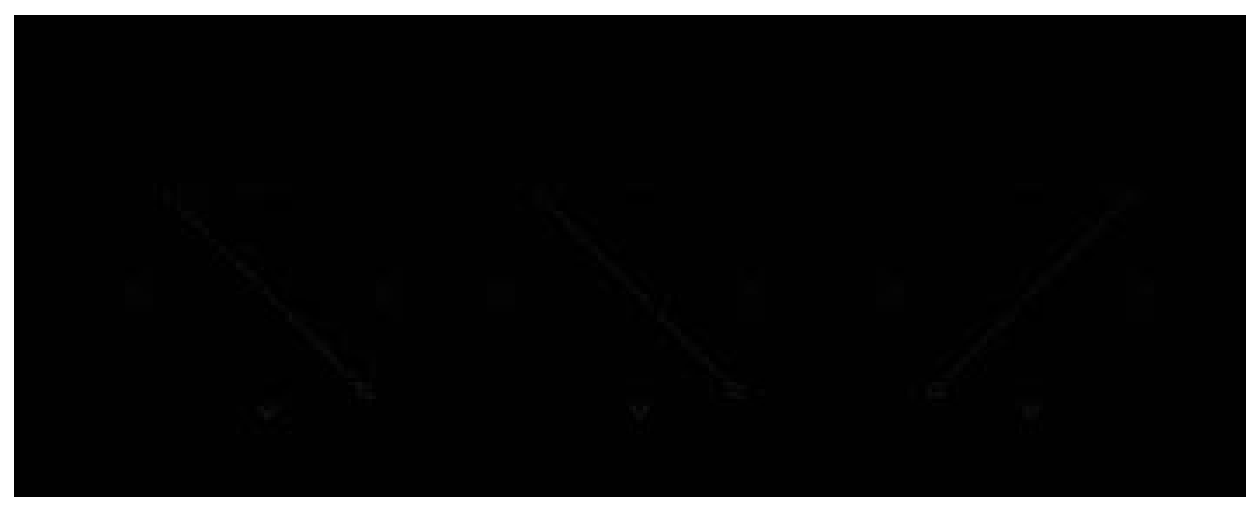

Figura 3. Caracterización real del funcionamiento del emprendimiento moral con relación a las clases sociales en las tres dimensiones analizadas.

\section{Conclusiones}

Los resultados de este trabajo sugieren que la ejecución de la función de emprendimiento moral está presente en la sociedad chilena. Sin embargo, parte de los estudios que ubican a la clase alta ejecutando dicha función o aquellos que entienden la clase baja en una posición más libertaria, no necesariamente consideran la distinción conceptual entre esfera pública y privada por una parte, y por otra no incorporan a la televisión como parte constitutiva de sus identidadesculturales.

En primer lugar, esta función se despliega en consideración a elementos de clase, y ámbitos de acción públicos y privados. Segundo, sólo en la esfera privada la clase alta tiene patrones conductuales que pueden ser relacionadosal emprendimiento moral. Esto, como sugiere el concepto de tradicionalismo ideológico, sucede porque miembros de dicho grupo social aceptan la modernización de ciertos procesos, en este caso el de la televisión, y elaboran estrategias de protección para instancias consideradas por ellos más valiosas, i.e. familia.Tercero, para el caso de las clases bajas, y retomando el concepto de conciencia dual sugerido por Fanon, la función de emprendimiento moral se vincula fuertemente a la esfera pública, como una estrategia de blanqueamiento (o de adaptación) de su discurso. Esto último, sin embargo, implica que dicha función tenga un carácter latente, por cuanto la televisión, no se somete a los valores que este grupo declara defender. C uarto, respecto a los efectos negativos de la televisión, como otra dimensión de la función de emprendimiento moral, las diferencias encontradas también indican que la clase baja está en mayor sintonía con dichos enunciados, ello porque la televisión es considerada como un aparato que influye 
directamente en la conducta de las personas, sin importar la mediación de otros elementos, entre los cuales por cierto se encuentra la propia reflexión que los sujetos puedan llevar a cabo.

Este trabajo plantea nuevas hipótesis de investigación para losámbitos macro y micro, las cuales finalmente permitirían conocer con mayor profundidad la relación identidad chilena/ televisión. Es de especial interés, conocer si la televisión está efectivamente clausurada a algunos de los valores que las clases bajas declaran. Por otra parte, es necesario ahondar en la investigación sobre qué mecanismos sociales estarían inhibiendo y/ o potenciando la función de emprendimiento moral en las esferas privadas, puesto que ello finalmente puede llevar a conocer cómo los factores culturales y estructurales están operando y son distribuidos en la sociedad chilena.

\section{$R$ eferencias}

A ranibar, P. (2001). A cercamiento conceptual a la situación adulto mayor en América Latina. Serie 21 Población y D esarrollo, CEPAL.

A tria, R . (2004). Estructura ocupacional, estructura social y clases sociales. N 0 96, Políticas Sociales, CEPAL.

Becker, H . (1963). O utsiders. Studies in the Sociology of D eviance. N uevaYork:T heFree Press. Berg, Axel (1998). Is sociological theory too grand for social mechanisms. En Peter H edstrom y R ichard Swedbergin (eds.), Social M echanisms.A nalytical A pproach to Social Theory. C ambridge: $C$ ambridge $U$ niversity Press.

Blofield, M . (2002). G uerra Santa. La izquierda y la derecha frente a los temas valóricos en C hile democrático. En M ireya D ávila y Claudio A. Fuentes (eds.), Promesas de cambio: Lalzquierday la D erecha en el C hile C ontemporáneo. Santiago:U niversitaria.

Bourdieu,P.(1997). Ladistinction. C ritique sociale du jugement. París. LesÉditionsde M inuit. — . (1998). Sur la télévision. París: Ed. R aisonsd'agir.

- . (2000). Poder, derecho y clasessociales Bilbao: D esclée de B rouwer.

Brunner, J.J. y C . C atalán. (1995). Televisión. Libertad, mercado y moral. Santiago:E ditorial LosAndes.

C astells, M anuel. (2005). G lobalización, desarrollo y democracia: C hile en el contexto mundial. Santiago: Fondo de Cultura Económico.

Dávila, M. (2002). La derecha y la socialdemocracia en Chile: principios y políticas públicas. En M ireya Dávila y C laudio A. Fuentes (eds.), Promesas de cambio: La Izquierda y laD erecha en el Chile C ontemporáneo. Santiago:U niversitaria.

D uarte, K . (2002). M undosjóvenes, mundos adultos: lo generacional y la reconstrucción de los puentes rotos en el Liceo. U na mirada desde la convivencia escolar. U Itima Década(Viña del M ar, C idpa), 16: 99-118.

Elias, N . (1994). The civilizing process: the history of mannersand stateformation and civilization. Oxford: Blackwell.

Fanon, F. (1971). Peau noire, masques blancs París. Seuil.

Germani, G. (1981). The sociology of modernization: studies on itshistoricaland theoretical aspects with special regard to the Latin A merican case. $\mathrm{N}$ ew B runswick, $\mathrm{N}$ ew Jersey: Transaction.

G offman, E. (1959). The presentation of self in everyday life. Garden C ity: D oubleday.

G offman,E. (9161). A sylumsessayson the social situation of mental patientsand other inmates Garden City: Anchor Books. 
Goode, E y N . Ben-Yehuda. (1999). M oral panics.The social construction of deviance.M alden, M A: Blacwell Publishers.

H abermas,..(1989). Thestructural transformation of the public sphere. C ambridge:T heM IT Press.

- .(1999). Between factsand norms. Contributionsto adiscourse theory of law and democracy. C ambridge:T he MIT Press.

H all, J., M .J. N eitz y M . Battani. (2003). Sociology on culture. Londres: R outledge, 2003. Josephson,W.L.(1997).Television violence and children's aggression:Testing the priming, social script, and disinhibition predictions. Journal of Personality and Social Psychology, 53: $882-890$

Joy, L. A., M . M . Kimball y M . L. Zabrack. (1986).Television and children's aggressive behavior. En T. M .W illiams (ed.), T he impact of television:A natural experiment in three communities (pp. 303-360). N ew York:A cademic Press.

Kirsch, H .W.(1977). Industrial developmentin a traditiona society: the conflict of entrepreneurship and modernization in Chile. G aisnville.

Larraín, J. (2001). La ldentidad C hilena. Santiago: Lom.

Lechner, N . (1999). D esafíos de un desar rollo humano: Individualizacion y capital social. Paper presentado como contribución al Foro Desarrollo y Cultura organizado por Science Po paraA samblea G eneral del Banco Interamericano de D esarrollo. París.

M arín, C . y R . C ordero, «Los M edio M asivosy lasTransformaciones de la Esfera Pública en C hile» Persona y SociedadVol. XIX N 으 233-258, 2005;Torche, F., «U nequal but fluid: social mobility in Chilein comparative perspective» A merican Sociological $R$ eview Vol. 70: 422-450, 2005.

M cN eely, C.L. (1997). Perceptions of the criminal justice system:Television Imagery and Public K nowledge in the U nited States. Journal of C riminal Justice and Popular Culture, 3(1): 1-20.

M olina, O. (2004). Siempre mañana y nunca mañamos. El círculo vicioso de la cultura oral. Santiago: Ediciones B.

M ontecino, S. (1996). D evenir de una traslación: de la mujer al género o de lo universal a lo particular. En Sonia M ontecino y Loreto R ebolledo (eds.), Concepto de género y desarrollo. Santiago: U niversidad de C hile, PIEG . 1996.

M orandé, P. (1984). Culturay modernización en A mérica Latina. C uadernos del Instituto de Sociología. Santiago U niversidad C atólica de C hile.

M oulian,T. (1997). Chile actual:A natomía de un mito. Santiago:A rcisy Lom.

O lavarría, J. (2000). De la identidad a la política: masculinidades y políticas públicas. A uge y ocaso de la familia nuclear patriarcal en el siglo XX. En José O lavarría y R odrigo Parrini (eds.), M asculinidad/es Identidad, sexualidad y familia. Santiago:, R ed de $M$ asculinidad-Flacso-U niversidad A cademia de H umanismo C ristiano.

0 ssa, M . (1996). Laidentidad pentecostal. Persona y Sociedad, 10(1).

O ssowski, S. (1963). C lass structure in the social consciousness. N uevaYork: Free Press of Glencoe.

Peppelenbos, L.P.(2005). The chilean miracle:Patrimonialism in a modern free market democracy. Thesis D issertation Wageningen U niversity.

PN U D . (1998). Desarrollo H umano en Chile. Santiago: PN U D.

PN U D . (2004). D esarrollo H umano en Chile. El Poder: ¿Paraquéy para quién? Santiago

R ovira, C.. (2005).T res grandes desafíos para los candidatos a la Presidencia de C hile. En www. asuntospublicos.org/ informe.php.

R ytina, S. (2000). Is occupational mobility declining in the U nited States? Social Forces, 78(4): 1227-76. 
Salazar, G. (1990). C hile, historiay bajo pueblo. Proposiciones, 19: 13.

- . (1991). The history of popular culture in C hile: Different paths. En K. A man y C. Parker, Popular culture in Chile. $R$ esistance and survival. Boulder:Westview Press

Salazar, G abriel y Julio Pinto. (1999). H istoria contemporánea de C hile I. Estado, legitimidad, ciudadanía Santiago: Lom.

Tironi, E. (1999). Lalrrupción de lasM asasy el M alestar de lasElites. Chile en el C ambio de Siglo. Santiago: Editorial G rijalbo.

T ironi, E., F. A güero y E.Valenzuela. (2001). C livajes políticos en C hile: perfil sociológico de los electores de Lagosy Lavín. $R$ evistaPerspectivas(D epartamento de Ingeniería Industrial, U niversidad de C hile), 5 (1): 73-87.

T ironi, E. y G. Sunkel. (1993). M odernización de las comunicaciones y democratización de lapolíticas. EstudiosPúblicos, 52: 215-246.

Torche, F. y G.Wormald. (2004). Stratification and mobility in C hile: Between ascription and achievement.Working D ocument 98, Social Policies Series, EC LAC.

Valdés,T. y X.Valdés. (editoras). (2005). Familia y vida privada. ¿Tranformaciones, tensiones, resistenciaso nuevos sentidos? Santiago:FLAC SO - C hile, C EDEM , U N FPA.

Variosautores. (2005). Conservadurismo y transgresión en Chile: $R$ eflexionessobre el mundo privado. Santiago: FLACSO - C hile, CEDEM .

Velasco,A. (2001). La Infelicidad. $R$ evistas Perspectivas (D epartamento de Ingeniería Industrial U niversidad de Chile), 5 (1): 31-40.

Weber, M . (1944). From M ax Weber: Essays on Sociology. Londres: R outledge y Kegan Paul.

W right, E. O. (1997). Class counts: Comparative studies in class analysis. Cambridge: Cambridge: U niversity Press.

W right M ills, C. (1956). The power elite. 0 xford: 0 xford Press. 